\title{
High-quality $a$-plane GaN grown with flow-rate modulation epitaxy on $r$-plane sapphire substrate
}

\author{
Jeng-Jie Huang, Tsung-Yi Tang, Chi-Feng Huang, C.C. Yang* \\ Graduate Institute of Electro-Optical Engineering and Department of Electrical Engineering, \\ National Taiwan University, 1, Roosevelt Road, Section 4, Taipei, Taiwan, ROC \\ Received 25 June 2007; accepted 5 February 2008 \\ Communicated by G.B. Stringfellow \\ Available online 16 February 2008
}

\begin{abstract}
We use the flow-rate modulation epitaxy (FME) technique to improve the crystal quality of a pit-free $a$-plane GaN (1 $1 \overline{2} 0)$ film grown on $r$-plane sapphire $\left(\begin{array}{llll}1 & 1 & 0 & 2\end{array}\right)$ substrate. With the FME technique, the width of the rocking curve in X-ray diffraction measurement is significantly reduced. Also, the surface roughness in either atomic-force-microscopy scanning or $\alpha$-step profiling is decreased. Here, the FME technique means to alternatively turn on and off the supply of Ga atoms, while $\mathrm{N}$ atoms are continuously supplied without changing the flow rate. Under the used growth conditions, the optimized FME parameters include the on/off periods at 10/10 s. During the period of closing the flow of trimethylgallium (TMGa), the continuous supply of nitrogen can lead to the formation of stoichiometry structure under the high-Ga growth condition, which is required for pit-free growth. Also, during this period, Ga atoms can further migrate to result in a flatter surface. Therefore, the crystal quality of the $a$-plane GaN sample can be improved.
\end{abstract}

(C) 2008 Elsevier B.V. All rights reserved.

Keywords: A1. Crystal morphology; A3. Metalorganic chemical vapor deposition; B1. Nitrides; B3. Light-emitting diodes

\section{Introduction}

Wurtzite III-nitrides have been widely studied because of their broad applications to light-emitting diodes and laser diodes. Currently, either the light-emitting diodes or laser diodes are mostly fabricated with $c$-plane $\left(\begin{array}{llll}0 & 0 & 0 & 1\end{array}\right)$ $\mathrm{InGaN} / \mathrm{GaN}$ quantum well (QW) structures. However, in a $c$-plane structure, large pyroelectric and piezoelectric fields exist along the $c$-axis with an internal electric field of about $1 \mathrm{MV} / \mathrm{cm}$ across an InGaN/GaN QW [1]. In this situation, the electrons and holes in the QW are spatially separated leading to the reduced recombination rate for emission and the red shift of emission wavelength. Such a behavior, called the quantum-confined stark effect (QCSE), represents one of the unsolved problems in utilizing III-nitride compounds for fabricating light-emitting devices $[2,3]$. In such a device, when a large injection current is applied, the

\footnotetext{
*Corresponding author. Tel.: + 886223657624 ; fax: + 886223652637.

E-mail address: ccy@cc.ee.ntu.edu.tw (C.C. Yang).
}

carrier screening of the QCSE can result in emission wavelength blue shift.

To solve the problem, non-polar nitride structures have been developed. In such a structure, the $\left[\begin{array}{llll}0 & 0 & 0 & 1\end{array}\right] c$-axis lies in the growth surface and hence no built-in field exists across a QW. The absence of the built-in field has been widely demonstrated in such a non-polar structure and light-emitting diodes have been fabricated with such a structure [4,5]. Recently, laser diodes based on non-polar InGaN/GaN QW structures have been demonstrated [6,7]. Non-polar $\mathrm{GaN}$ can be grown with several configurations, including $a$-plane $\mathrm{GaN}$ on $r$-plane sapphire substrate [5], $m$-plane $\mathrm{GaN}$ on $m$-plane $6 \mathrm{H}$-SiC substrate [8] or $\gamma-\mathrm{LiAlO}_{2}$ substrate [4], and $m$-plane $\mathrm{GaN}$ on $m$-plane GaN substrate [9]. In spite of all those accomplishments, so far, the emission efficiencies of the non-polar-based light-emitting devices are still lower than those of their counterparts based on $c$-plane structures. The lower efficiency is attributed to the higher threading dislocation density and other defects. Hence, the improvement of crystal quality of non-polar nitrides still needs more efforts. 
Because of the different growth rates along different crystal axes, undulated or trench structures exist on a nonpolar GaN surface [10]. In the case of $a$-plane $\mathrm{GaN}$ grown on $r$-plane sapphire substrate, the trenches are along the $c$-axis. For high-quality device fabrication, the 1-D epitaxial lateral overgrowth (ELOG) technique has been used with the stripes perpendicular to the trenches for improving the crystal quality. In the case of growing $a$-plane GaN on $r$-plane sapphire substrate, the dielectric mask stripes in ELOG are along the $m$-axis for first enhancing the growth rate in the this direction. After filling up the gaps between the stripes, the high $c$-axis growth rate eventually results in the coalescence growth leading to high crystal quality [11]. Normally, a lower chamber pressure, a higher growth temperature, and a lower $\mathrm{V} / \mathrm{III}$ ratio (high-Ga condition) are the three key factors for growing high-quality, pit-free $a$-plane $\mathrm{GaN}$ on $r$-plane sapphire substrate with metalorganic chemical vapor deposition (MOCVD) $[10,12]$. Under the above growth conditions, the full-width at half-maximum (FWHM) of the rocking curve in X-ray diffraction (XRD) measurement ranges from 800 through 1000 arcsec $[10,13,14]$. Better results with the rocking curve FWHM lower than 700 arcsec were reported in $a$-plane $\mathrm{GaN}$ films thicker than $10 \mu \mathrm{m}$ (even up to several $10 \mathrm{~mm}$ ) [12,15]. Such a thick GaN template is of course impractical for device fabrication.

In this paper, we report the improved pit-free $a$-plane GaN crystal quality on $r$-plane sapphire substrate based on a flow-rate modulation epitaxy (FME) method. The FME method has been used for growing high-quality AlN films on $\mathrm{SiC}$ substrate by alternating the supplies of group-III and group-V sources [16]. With the alternating supplies of the two kinds of source, the growth rate is quite low $(\sim 300 \mathrm{~nm} / \mathrm{h})$ that might not be practical in application. In our study, we alternatively switch on and off the Ga atom supply with continuous nitrogen supply. Under such a growth condition, the rocking curve FWHM in XRD measurement is significantly reduced (down to 679 arcsec) and the surface roughness in atomic-force-microscopy (AFM) scanning or $\alpha$-step profiling is significantly decreased. With our FME technique, the growth rate can be as high as $2.28 \mu \mathrm{m} / \mathrm{h}$ and the required thickness of the $a$-plane GaN films for showing high crystalline quality is only $4 \mu \mathrm{m}$, which is quite practical in application. It is believed that with such a higher-quality $a$-plane $\mathrm{GaN}$ template, after the subsequent ELOG process, the fabricated light-emitting devices can be more efficient. In Section 2 of this paper, we introduce the sample growth and characterization conditions. Then, the sample characterization results are reported in Section 3. Finally, the results are discussed and concluded in Section 4.

\section{Sample growth conditions}

Totally nine $(11 \overline{2} 0) a$-plane GaN thin-film samples are grown on (1 $1 \overline{1} 02) r$-plane sapphire substrate of \pm 2 in orientation inaccuracy. The substrate is thermally treated with ambient hydrogen at $1120^{\circ} \mathrm{C}$ before the growth in a MOCVD reactor (Veeco P75). The growth procedure is the same as the conventional two-step growth of $c$-plane GaN except that an FME growth technique is used. Trimethylgallium $(\mathrm{TMGa})$ and ammonia $\left(\mathrm{NH}_{3}\right)$ are used as $\mathrm{Ga}$ and $\mathrm{N}$ sources, respectively. Hydrogen is used as the carrier gas. A GaN buffer layer of $20 \mathrm{~nm}$ in thickness is first grown at $530{ }^{\circ} \mathrm{C}$ with the chamber pressure at 500 Torr and the V/III ratio at about 5200. After the growth of the buffer layer, the substrate temperature is ramped to $1100^{\circ} \mathrm{C}$ for depositing the $a$-plane GaN epitaxial layer with the FME method. The FME growth is implemented by alternatively opening and closing the $\mathrm{TMGa}$ valve with different durations while the supply of $\mathrm{NH}_{3}$ is unchanged. Different FME growth conditions for different samples are listed in Tables 1 and 2. The chamber pressure for GaN growth is 50 Torr and the $\mathrm{V} / \mathrm{III}$ ratio is between 250 and 300 . The $\mathrm{GaN}$ growth rate is controlled by the flow rate of TMGa. The thickness of the $a$-plane GaN thin film in each sample is around $4 \mu \mathrm{m}$. High-resolution XRD with a Bede D1 system, AFM with a DI Dimension 3100 system, and $\alpha$-step profiling with a Veeco Dektak 6M system are used for characterizing the grown samples.

\section{Sample characterization results}

The grown samples are then examined with XRD $\omega-2 \theta$ scans with the X-ray incident plane parallel to the $c$-axis of

Table 1

Crystal quality parameters of the samples grown under different conditions of the FME growth

\begin{tabular}{llll}
\hline Sample No & $\begin{array}{l}\text { Flow } \\
\text { modulation } \\
\text { on/off (s/s) }\end{array}$ & $\begin{array}{l}\text { Rocking curve } \\
\text { FWHM } \\
\text { (arcsec) }\end{array}$ & $\begin{array}{l}\text { Surface } \\
\text { roughness (nm) }\end{array}$ \\
\hline A & always on & 984 & 12.7 \\
B & $10 / 10$ & 679 & 4.7 \\
C & $6 / 6$ & 857 & 5.6 \\
D & $15 / 15$ & 757 & 6.2 \\
E & $6 / 10$ & 860 & 5.0 \\
F & $10 / 6$ & 777 & 5.4 \\
G & $15 / 10$ & 803 & 6.3 \\
\hline
\end{tabular}

The surface roughness data are obtained from AFM scanning over an area of $10 \mu \mathrm{m} \times 10 \mu \mathrm{m}$ in dimension.

Table 2

Crystal quality parameters of the samples grown under different conditions of growth rate with a fixed flow modulation condition (on/ off at $10 / 10 \mathrm{~s})$

\begin{tabular}{lllll}
\hline Sample No & $\begin{array}{l}\text { Flow } \\
\text { modulation } \\
\text { on/off }(\mathrm{s} / \mathrm{s})\end{array}$ & $\begin{array}{l}\text { Growth } \\
\text { rate }(\mu \mathrm{m} / \mathrm{h})\end{array}$ & $\begin{array}{l}\text { Rocking } \\
\text { curve } \\
\text { FWHM } \\
(\operatorname{arcsec})\end{array}$ & $\begin{array}{l}\text { Surface } \\
\text { roughness } \\
(\mathrm{nm})\end{array}$ \\
\hline $\mathrm{B}$ & $10 / 10$ & 2.28 & 679 & 4.7 \\
$\mathrm{H}$ & $10 / 10$ & 2.37 & 745 & 6.0 \\
$\mathrm{I}$ & $10 / 10$ & 2.17 & 730 & 4.5 \\
\hline
\end{tabular}


the a-plane GaN films. The scan pattern of sample B (see Table 1) is shown in Fig. 1. The peak of ( $111 \overline{2} 0) \mathrm{GaN}$ at $2 \theta-57.7^{\circ}$ confirms the growth of $a$-plane GaN. With this scanning scale, the XRD patterns of all the samples look almost the same. The growth conditions and characterization results of various $a$-plane $\mathrm{GaN}$ samples are summarized in Table 1. The data of XRD rocking curve are obtained from the high-resolution XRD measurement on the $(11 \overline{2} 0)$ plane. The surface roughness data are obtained from AFM scanning over an area of $10 \mu \mathrm{m} \times 10 \mu \mathrm{m}$ in dimensions. In Table 1 , one can see that both the FWHM of XRD rocking curve and the surface roughness of sample A, which is grown with the conventional method, i.e. without using the FME technique, are the largest among the listed seven samples in Table 1. Such characterization results are commonly seen in literature $[10,13,14]$. In contrast, under the FME growth conditions, the FWHMs of the XRD rocking curves and the surface roughness levels become smaller. In other words, the crystalline quality is improved with this method. In particular, when the on/off durations are $10 / 10 \mathrm{~s}$ to give sample $B$, both parameters reach the minimum values at $679 \operatorname{arcsec}$ and $4.7 \mathrm{~nm}$. In this situation, the FWHM of the rocking curve represents one of the lowest values ever

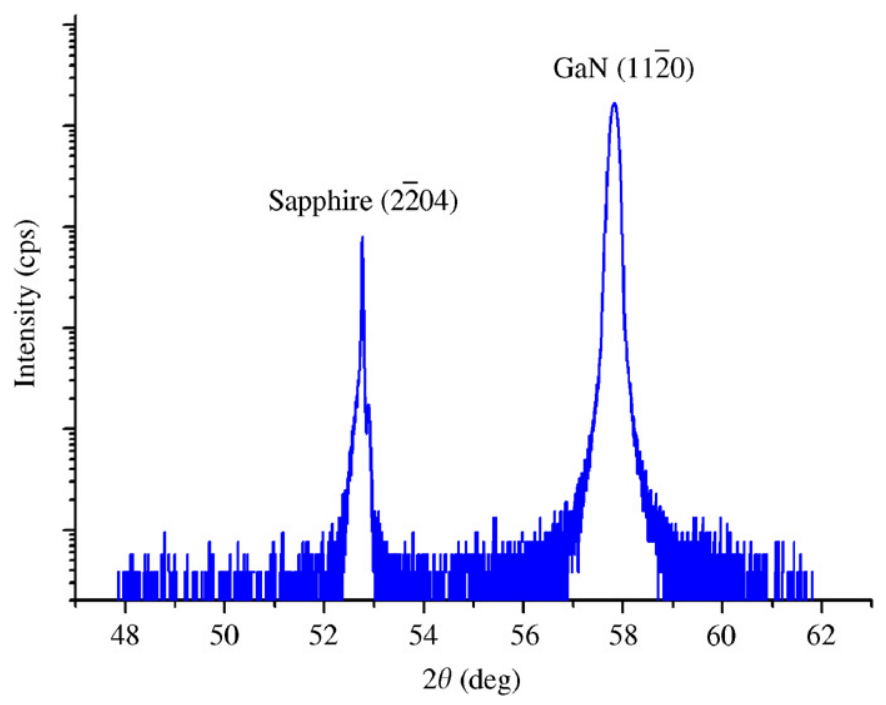

Fig. 1. XRD pattern of sample B. reported in literature $[12,15]$. From Table 1, one can see that, except samples A and B, the sizes of the rocking curve FWHM and the levels of surface roughness among different samples are not strongly correlated. Also, except the optimized condition of sample B, the surface roughness is weakly dependent on the modulation flow condition. However, the rocking curve FWHM follows certain rules of flow modulation. With the comparisons between samples B and F, and between D and G, one can see that the increase of the Ga 'off' duration, while keeping the same and longer 'on' duration, leads to a smaller rocking curve FWHM and slightly smoother surface morphology. For either 'on' or 'off' duration, $10 \mathrm{~s}$ is the optimized period.

In Table 2, we compare the characterization results of three samples of different growth rates with a fixed FME condition same as that of sample B. By slightly lowering the growth rate, as the case of sample I, the surface roughness is slightly decreased that can be due to the measurement inaccuracy. However, the rocking curve FWHM is significantly increased. On the other hand, by increasing the growth rate, neither XRD result nor surface roughness is improved. The growth rate of $2.28 \mu \mathrm{m} / \mathrm{h}$ with the $10 / 10 \mathrm{~s}$ for the on/of durations is the optimized condition.

As mentioned earlier, before device fabrication, the 1-D ELOG technique is usually used for further improving the overall crystal quality. Therefore, surface roughness due to the trench pattern on $a$-plane GaN needs to be minimized for the subsequent better-quality regrowth and hence device fabrication. To further understand the surface morphologies of the grown samples, in Fig. 2, we compare the optical images of samples A (a) and B (b) under a Normaski optical microscope with $1000 \times$ magnification. Here, one can see that the trench pattern of sample B is not as clear as that of sample A implying that the global surface quality of sample B is also better.

The AFM images of $10 \mu \mathrm{m} \times 10 \mu \mathrm{m}$ in dimensions (a) and their line-scan spatial frequency distributions (b) of samples A and B are shown in Figs. 3 and 4, respectively. From the color contrast, one can easily see the less-rough surface of sample B ( $4.7 \mathrm{~nm}$ roughness on sample B versus $12.7 \mathrm{~nm}$ roughness on sample $\mathrm{A}$ ). The trenches are along the [ $\left.\begin{array}{llll}0 & 0 & 1 & 1\end{array}\right]$ direction. Also, from the spatial frequency
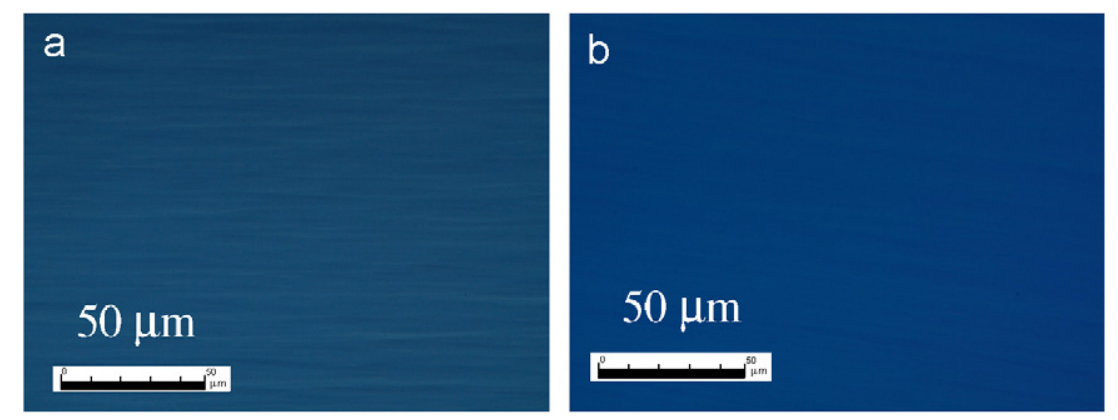

Fig. 2. Images of samples A (a) and B (b) under a Normaski optical microscope with $1000 \times$ magnification. 
a

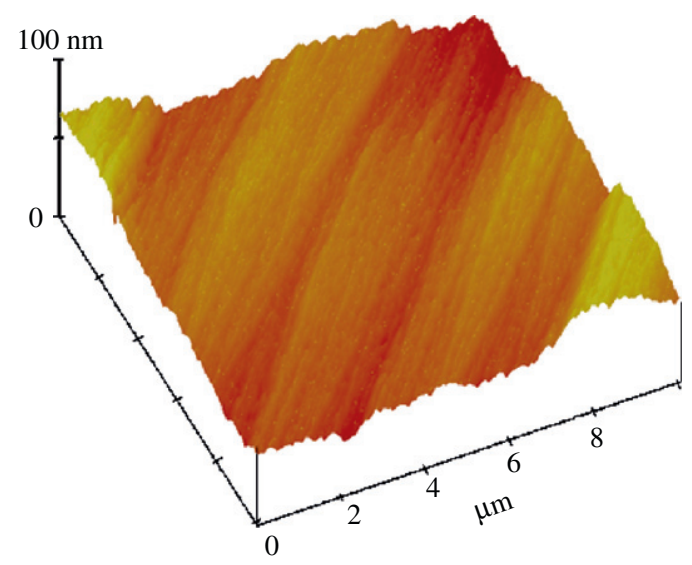

b

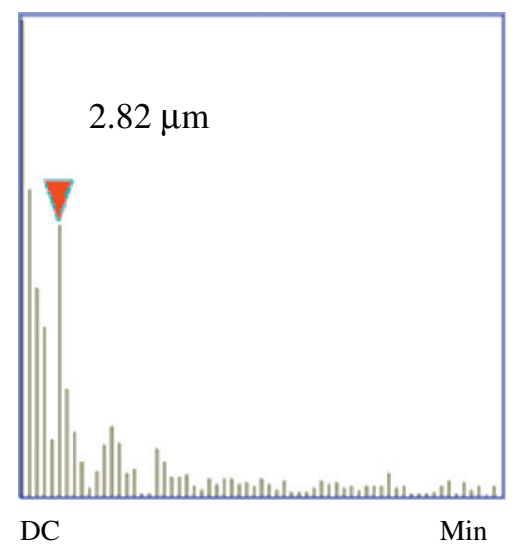

Fig. 3. AFM image (a) $(10 \mu \mathrm{m} \times 10 \mu \mathrm{m}$ in dimensions $)$ and its line-scan spatial frequency distribution (b) of sample A.

distributions when scanning in the direction perpendicular to the trenches, we can read the major trench periods of 2.82 and $4.10 \mu \mathrm{m}$ on samples $\mathrm{A}$ and $\mathrm{B}$, respectively. The larger period in sample B implies the smoother surface of this sample.

Next, to examine the larger-scale surface roughness, we use an $\alpha$-step profiler to scan the sample surfaces in the direction perpendicular to the trenches. Fig. 5(a) shows the line-scan profiles of samples A and B over a length of $100 \mu \mathrm{m}$. The maximum-to-minimum heights of samples A and $B$ are 85 and $62 \mathrm{~nm}$, respectively, that once again indicates the smoother surface of sample B. In Figs. 5(b) and (c), we show the spatial frequency distributions of the line-scan profiles in Fig. 5(a) by taking the Fourier transformations for samples A and B, respectively. Here, one can see the sub-micron surface variations. Basically, the sub-micron spatial-frequency features of the two samples are about the same. Both of them have the features at around 0.06 and $0.11 \mu \mathrm{m}$ in period.

\section{Discussions and conclusions}

Normally, lower pressure, higher temperature, and lower $\mathrm{V} / \mathrm{III}$ ratio are the three key factors for growing pit-free a

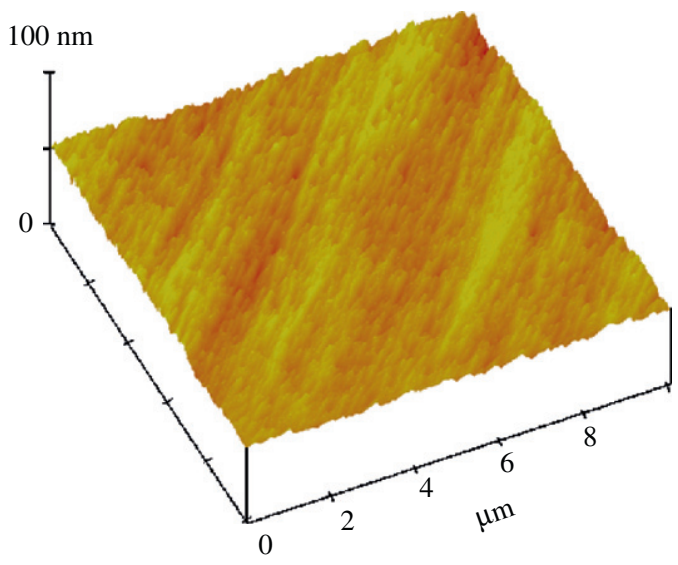

b

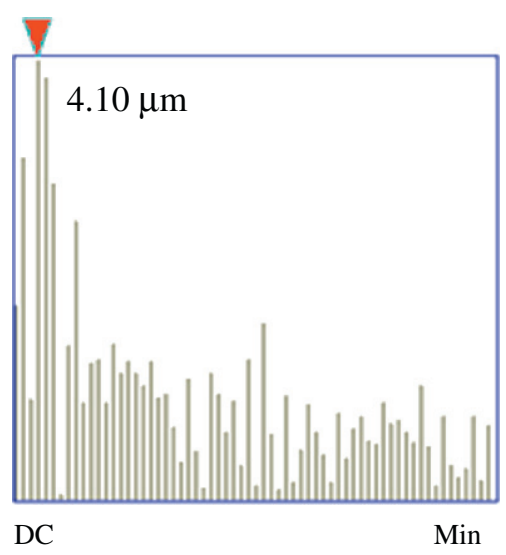

Fig. 4. AFM image (a) $(10 \mu \mathrm{m} \times 10 \mu \mathrm{m}$ in dimensions $)$ and its line-scan spatial frequency distribution (b) of sample B.

$a$-plane GaN on $r$-plane sapphire substrate. By considering these three factors, we were able to grow pit-free $a$-plane $\mathrm{GaN}$ (sample A) with our MOCVD reactor. By using the FME technique, the quality of $a$-plane $\mathrm{GaN}$ has been further improved (sample B and others). As mentioned earlier that for growing pit-free $a$-plane $\mathrm{GaN}$, the V/III ratio needs to be reduced. In such a high-metal growth condition, nitrogen vacancies can be easily generated in the crystal hindering the further improvement of crystal quality [17]. During the period of closing the flow of TMGa, under the high-temperature condition, the Ga atoms can further migrate to achieve a better 2-D growth result and hence a smaller surface roughness. Meanwhile, in this period, the continuous supply of nitrogen can reduce the nitrogen vacancy density leading to a better-quality stoichiometry structure. The length of this Ga "off" period is quite critical for high-quality result, as can be seen in the comparisons between samples $\mathrm{B}$ and $\mathrm{F}$ and between samples D and G. In these comparisons, one can see that a longer Ga "off" period for the aforementioned processes leads to a smaller rocking curve FWHM and a smaller surface roughness. The optimized $10 / 10 \mathrm{~s}$ condition simply means that the deposition of high-metal atomic layers during the 10-s TMGa flow period plus the atomic 
a

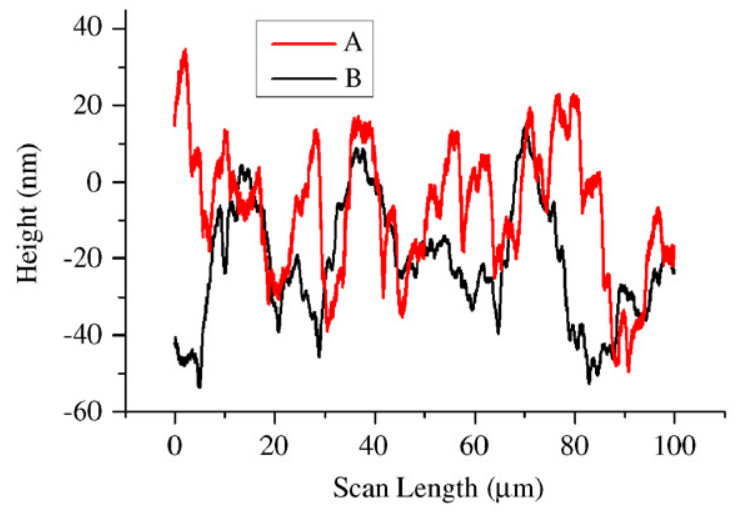

b

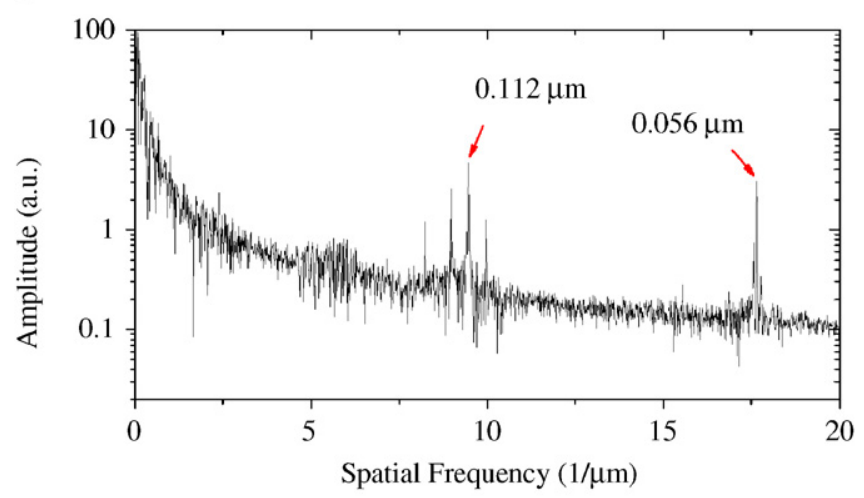

C

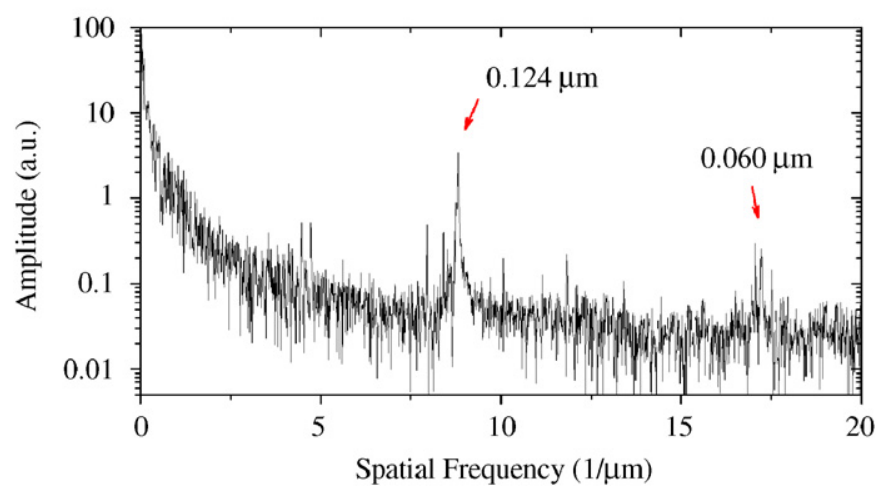

Fig. 5. Scanning results of sample surface roughness with an $\alpha$-step profiler: (a) Line-scan profiles along the direction perpendicular to the surface trenches of samples A and B; (b) The spatial frequency distribution of sample A; (c) The spatial frequency distribution of sample B.

rearrangement during another 10-s Ga closing period can lead to the best crystal quality under the current growth conditions.

In summary, we have used the FME technique to

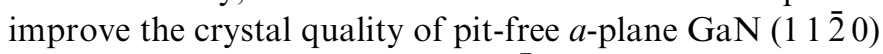
films grown on $r$-plane sapphire ( $\left.\begin{array}{llll}1 & \overline{1} & 0 & 2\end{array}\right)$ substrate. With the FME technique, the rocking curve FWHM was significantly reduced down to $679 \mathrm{arcsec}$, which is one of the smaller values in a thin $a$-plane GaN film (less than a few microns) grown on $r$-plane sapphire substrate. By using this technique, the surface roughness was also significantly decreased down to $4.7 \mathrm{~nm}$ within a $10 \mu \mathrm{m} \times 10 \mu \mathrm{m}$ area. The FME technique meant to alternatively turn on and off the supply of $\mathrm{Ga}$ atoms while $\mathrm{N}$ atoms were continuously supplied. Under the used growth conditions, the optimized FME parameters included the on/off periods at 10/10 s.

\section{Acknowledgments}

This research was supported by the National Science Council, The Republic of China, under the grant of NSC 95-2120-M-002-012 and NSC 95-2221-E-002-287, and by the US Air Force Scientific Research Office under the contract AOARD-06-4052.

\section{References}

[1] S. Ghosh, P. Misra, H.T. Grahnb, B. Imer, S. Nakamura, S.P. DenBaars, J.S. Speck, J. Appl. Phys. 98 (2005) 026105.

[2] S.F. Chichibu, A.C. Abare, M.S. Minsky, S. Keller, S.B. Fleischer, J.E. Bowers, E. Hu, U.K. Mishra, L.A. Coldren, S.P. DenBaars, T. Sota, Appl. Phys. Lett. 73 (1998) 2006.

[3] C.K. Choi, Y.H. Kwon, B.D. Little, G.H. Gainer, J.J. Song, Y.C. Chang, S. Keller, U.K. Mishra, S.P. DenBaars, Phys. Rev. B. 64 (2001) 245339.

[4] P. Waltereit, O. Brandt, A. Trampert, H.T. Grahn, J. Menniger, M. Ramsteiner, M. Reiche, K.H. Ploog, Nature 406 (2000) 865.

[5] A. Chitnis, C. Chen, V. Adivarahan, M. Shatalov, E. Kuokstic, V. Mandavilli, J. Yang, M.A. Khan, Appl. Phys. Lett. 84 (2004) 3663.

[6] M.C. Schmidt, K.C. Kim, R.M. Farrell, D.F. Feezell, D.A. Cohen, M. Saito, K. Fujito, J.S. Speck, S.P. DenBaars, S. Nakamura, Jpn. J. Appl. Phys. 46 (2007) L190.

[7] D.F. Feezell, M.C. Schmidt, R.M. Farrell, K.C. kim, M. Saito, K. Fujito, D.A. Cohen, J.S. Speck, S.P. DenBaars, S. Nakamura, Jpn. J. Appl. Phys. 46 (2007) L284.

[8] B. Imer, F. Wu, M.D. Craven, J.S. Speck, S.P. DenBaars, Jpn. J. Appl. Phys. 45 (2006) 8644.

[9] A. Chakaraborty, B.A. Haskell, S. Keller, J.S. Speck, S.P. DenBaars, S. Nakamura, U.K. Mishra, Jpn. J. Appl. Phys. 44 (2005) L173.

[10] X. Ni, Y. Fu, Y.T. Moon, N. Biyikli, H. Morkoç, J. Crystal Growth 290 (2006) 66.

[11] M.D. Craven, S.H. Lim, F. Wu, J.S. Speck, S.P. DenBaars, Appl. Phys. Lett. 81 (2002) 1201.

[12] M. Araki, N. Mochimizo, K. Hoshino, K. Tadatomo, Jpn. J. Appl. Phys. 46 (2007) 555.

[13] D. Li, H. Chen, H.B. Yu, Y.J. Han, X.H. Zheng, Q. Huang, J.M. Zhou, J. Crystal Growth 263 (2004) 76.

[14] M.D. Craven, S.H. Lim, F. Wu, J.S. Speck, S.P. DenBaars, Appl. Phys. Lett. 81 (2002) 469.

[15] T. Paskova, R. Kroeger, S. Figge, D. Hommel, V. Darakchieva, B. Monemar, E. Preble, A. Hanser, N.M. Williams, M. Tutor, Appl. Phys. Lett. 89 (2006) 051914.

[16] T. Akasaka, Y. Kobayashi, T. Makimoto, Appl. Phys. Lett. 90 (2007) 121919.

[17] P. Kozodoy, S. Keller, S.P. DenBaars, U.K. Mishra, J. Crystal Growth 195 (1998) 265. 